\title{
Влияние параметров квантовой ямы на спектр двумерных плазмонов в гетероструктурах $\mathrm{HgTe} / \mathrm{CdHgTe}$
}

\author{
(C) В.Я. Алешкин ${ }^{1,2}$, А.А. Дубинов ${ }^{1,2}$ \\ ${ }^{1}$ Институт фризики микроструктур Российской академии наук, \\ 603950 Нижний Новгород, Россия \\ ${ }^{2}$ Нижегородский государственный университет им. Н.И. Лобачевского, \\ 603950 Нижний Новгород, Россия \\ E-mail: aleshkin@ipmras.ru \\ Поступила в Редакцию 12 апреля 2021 г. \\ В окончательной редакции 19 апреля 2021 г. \\ Принята к публикации 19 апреля 2021 г.
}

\begin{abstract}
Теоретически изучено влияние параметров квантовой ямы и пространственной дисперсии электронной поляризуемости на зависимость энергии двумерных плазмонов от волнового вектора в узкозонных квантовых ямах $\mathrm{CdHgTe}$ (ширина запрещенной зоны 35 мэВ). Показано, что при энергиях > 20 мэВ закон дисперсии двумерных плазмонов близок к линейному. Учет конечной ширины квантовой ямы уменьшает фазовую скорость плазмона. Этот эффект увеличивается с ростом доли кадмия в слое квантовой ямы при сохранении ширины запрещенной зоны и с уменьшением концентрации носителей заряда в ней.
\end{abstract}

Ключевые слова: двумерные плазмоны, квантовые ямы $\mathrm{HgTe}$.

DOI: $10.21883 /$ FTP.2021.11.51549.45

\section{1. Введение}

Хорошо известно, что для волновых векторов $\mathbf{q}$, много бо́льших $\omega / c(\omega-$ частота, $c-$ скорость света в вакууме), частота двумерного (2D) плазмона пропорциональна квадратному корню из его волнового вектора [1]. Однако эта зависимость справедлива только в том случае, когда пространственная дисперсия поляризуемости 2D электронного газа не существенна [1] (т. е. q много меньше фермиевского волнового вектора электронов). Кроме того, при нахождении закона дисперсии двумерного плазмона часто полагают, что длина волны плазмона много больше ширины квантовой ямы. Обычно эти условия хорошо выполняются для плазмонов с частотами до 1 ТГц в системах с квантовыми ямами $\mathrm{GaAs}$ [2]. Однако оба эти условия могут нарушаться при рассмотрении межзонных двумерных плазмонов, энергия которых превышает ширину запрещенной зоны квантовой ямы HgTe [3], которая несколько превышает энергию продольного оптического фонона в $\mathrm{CdHgTe}$ (21 мэВ). Интерес к таким плазмонам возник в связи с возможностью их стимулированной генерации в узкозонных квантовых ямах HgTe [3]. Заметим, что учет пространственной дисперсии поляризуемости электронного газа в графене приводит к зависимости частоты плазмона от его волнового вектора, сильно отличающейся от корневой в области больших частот [4]. Однако, если при рассмотрении плазмонов в графене толщиной графена можно пренебречь по сравнению с длиной волны 2D плазмона, то для квантовых ям HgTe ширина квантовой ямы не всегда мала по сравнению с длиной волны плазмона. В связи с этим возникает вопрос о том, как изменится закон дисперсии 2D плазмона при учете конечной ширины квантовой ямы. Этот вопрос является важным, например, при вычислении темпа рекомбинации неравновесных носителей с испусканием плазмона или при расчете коэффициента усиления плазмонов в условиях инверсной населенности зон.

Настоящая работа посвящена теоретическому изучению влияния пространственной дисперсии восприимчивости и параметров квантовых ям, в том числе и ширины, на закон дисперсии двумерных плазмонов в гетероструктурах $\mathrm{HgTe} / \mathrm{CdHgTe} \mathrm{с} \mathrm{квантовыми} \mathrm{ямами.}$

\section{2. Метод расчета спектра двумерных плазмонов}

Для нахождения закона дисперсии плазмона будем использовать квазистатическое приближение, в котором пренебрегается эффектами запаздывания [5]. Кроме того, для простоты будем полагать диэлектрическую проницаемость $\kappa$ одинаковой в барьерах и ямах. Поскольку мы интересуемся плазмонами с частотой, большей частоты оптических фононов, будем полагать величину диэлектрической проницаемости равной ее высокочастотному значению. Пусть вдоль квантовой ямы распространяется волна потенциала с волновым вектором q и частотой $\omega$ :

$$
\begin{aligned}
\varphi(\mathbf{r}, t)= & \varphi(z) \exp (i \mathbf{q r}-i \omega t+\alpha t) \\
& +\varphi^{*}(z) \exp (-i \mathbf{q} \mathbf{r}+i \omega t+\alpha t)
\end{aligned}
$$

где $\alpha>0$ - бесконечно малая величина, необходимая для правильного обхода полюсов, возникающих при вычислении поляризуемости электронного газа (см., например, получение формулы Линхарда в книге [6]). Ось $z$ выбрана в качестве нормали к квантовой яме. Представим волновую функцию электрона в квантовой 
яме в отсутствие потенциала в виде $\psi_{\mathbf{k}, s}^{0}(z) \exp (i \mathbf{k r}) / \sqrt{S}$, где индекс $s$ описывает и спиновое состояние электрона, и номер подзоны размерного квантования, $S$ - площадь квантовой ямы, $\mathbf{r}-$ радиус-вектор, $\mathbf{k}-$ волновой вектор электрона в квантовой яме. Первая поправка по теории возмущений к волновой функции электрона, возникающая из-за потенциала (1), есть

$$
\begin{gathered}
\left.\psi_{\mathbf{k}, s}^{(1)}(z, t)=\sum_{s^{\prime}} b_{s, s^{\prime}}(\mathbf{k}+\mathbf{q}, t) \psi_{\mathbf{k}+\mathbf{q}, s^{\prime}}(z) \exp [i(\mathbf{k}+\mathbf{q}) \mathbf{r})\right] \\
+c_{s, s^{\prime}}(\mathbf{k}-\mathbf{q}, t) \psi_{\mathbf{k}-\mathbf{q}, s^{\prime}}(z) \exp [i(\mathbf{k}-\mathbf{q}) \mathbf{r}]
\end{gathered}
$$

где

$$
\begin{aligned}
b_{s, s^{\prime}}(\mathbf{k} & +\mathbf{q}, t) \\
= & -e \frac{\exp \left[-i \omega t-i \varepsilon_{s}(\mathbf{k}) t / h+i \varepsilon_{s^{\prime}}(\mathbf{k}+\mathbf{q}) t / h+\alpha t\right]}{\varepsilon_{s}(\mathbf{k})+h \omega-\varepsilon_{s^{\prime}}(\mathbf{k}+\mathbf{q})+i h \alpha} \\
& \times \int d z \psi_{\mathbf{k}+\mathbf{q}, s^{\prime}}^{*}(z) \varphi(z) \psi_{\mathbf{k}, s}(z) \\
c_{s, s^{\prime}}(\mathbf{k}-\mathbf{q}) & \\
= & -e \frac{\exp \left[i \omega t-i \varepsilon_{s}(\mathbf{k}) t / h+i \varepsilon_{s^{\prime}}(\mathbf{k}-\mathbf{q}) t / h+\alpha t\right]}{\varepsilon_{s}(\mathbf{k})-h \omega-\varepsilon_{s^{\prime}}(\mathbf{k}-\mathbf{q})+i h \alpha} \\
& \times \int d z \psi_{\mathbf{k}-\mathbf{q}, s^{\prime}}^{*}(z) \varphi^{*}(z) \psi_{\mathbf{k}, s}(z)
\end{aligned}
$$

$e$ - заряд электрона, $\varepsilon_{s}(\mathbf{k})$ - энергия электрона с волновым вектором $\mathbf{k}$ в $s$-й подзоне, $h$ - постоянная Планка. Плотность заряда, вызванная добавкой к волновой функции (2), есть

$$
\begin{aligned}
\rho(z, t)= & -e \sum_{k, s}\left(\left|\psi_{\mathbf{k}, s}^{(0)}(z, t)+\psi_{\mathbf{k}, s}^{(1)}(z, t)\right|^{(2)}\right. \\
& \left.-\left|\psi_{\mathbf{k}, s}^{(0)}(z, t)\right|^{(2)}\right) f_{s}(\mathbf{k}),
\end{aligned}
$$

где $f_{s}(\mathbf{k})$ - функция распределения электронов в $s$-й подзоне. Используя равенства (2)-(4), выражение (5) можно представить в виде

$$
\begin{aligned}
& \rho(z, t)=e^{2} \sum_{\mathbf{k}, s, s^{\prime}} \psi_{\mathbf{k}, s}^{*}(z) \psi_{\mathbf{k}+\mathbf{q}, s^{\prime}}(z) \\
& \times \frac{\exp (i \mathbf{q r}-i \omega t) f_{s}(\mathbf{k})}{\varepsilon_{s}(\mathbf{k})+h \omega-\varepsilon_{s^{\prime}}(\mathbf{k}+\mathbf{q})+i h \alpha} \\
& \times \int d z \psi_{\mathbf{k}, s^{\prime}}^{*}(z) \varphi(z) \psi_{\mathbf{k}, s}(z)-e^{2} \sum_{\mathbf{k}, s, s^{\prime}} \psi_{\mathbf{k}-\mathbf{q}, s^{\prime}}^{*}(z) \psi_{\mathbf{k}+\mathbf{q}, s^{\prime}}(z) \\
& \times \frac{\exp (i \mathbf{q r}-i \omega t) f_{s}(\mathbf{k})}{\varepsilon_{s}(\mathbf{k})-h \omega-\varepsilon_{s^{\prime}}(\mathbf{k}-\mathbf{q})-i h \alpha} \\
& \times \int d z \psi_{\mathbf{k}-\mathbf{q}, s^{\prime}}(z) \varphi(z) \psi_{\mathbf{k}, s}^{*}(z)+c . c .,
\end{aligned}
$$

где символ с.с. обозначает комплексно сопряженное слагаемое.
Из формулы (6) видно, что плотность заряда можно разбить на две части, соответствующие $\varphi(z)$ и $\varphi^{*}(z)$, которые можно рассматривать отдельно. По этой причине далее мы будем рассматривать в потенциале (1) и плотности заряда (6) слагаемые, пропорциональные $\varphi(z)$. Перепишем формулу (6) в более удобном виде:

$$
\begin{aligned}
& \rho(\mathbf{r})=\frac{e^{2} \exp (i \mathbf{q} \mathbf{r}-i \omega t)}{S} \\
& \times \sum_{\mathbf{k}, s, s^{\prime}} \frac{\psi_{\mathbf{k}, s}^{*}(z) \psi_{\mathbf{k}+\mathbf{q}, s^{\prime}}(z)\left(f_{s}(\mathbf{k})-f_{s^{\prime}}(\mathbf{k}+\mathbf{q})\right)}{\varepsilon_{s}(\mathbf{k})+h \omega-\varepsilon_{s^{\prime}}(\mathbf{k}+\mathbf{q})+i h \alpha} \\
& \quad \times \int d z^{\prime} \psi_{\mathbf{k}+\mathbf{q}, s^{\prime}}^{*}\left(z^{\prime}\right) \varphi\left(z^{\prime}\right) \psi_{\mathbf{k}, s}\left(z^{\prime}\right) .
\end{aligned}
$$

Используя (7), уравнение Пуассона можно представить в виде

$$
\begin{aligned}
& -q^{2} \varphi(z)+\frac{d^{2}}{d z^{2}} \varphi(z)=-\frac{4 \pi}{\kappa} \frac{e^{2} \exp (i \mathbf{q r}-i \omega t)}{S} \\
& \quad \times \sum_{\mathbf{k}, s, s^{\prime}} \frac{\psi_{\mathbf{k}, s}^{*}(z) \psi_{\mathbf{k}+\mathbf{q}, s^{\prime}}(z)\left(f_{s}(\mathbf{k})-f_{s^{\prime}}(\mathbf{k}+\mathbf{q})\right)}{\varepsilon_{s}(\mathbf{k})+h \omega-\varepsilon_{s^{\prime}}(\mathbf{k}+\mathbf{q})+i h \alpha} \\
& \quad \times \int d z^{\prime} \psi_{\mathbf{k}+\mathbf{q}, s^{\prime}}^{*}\left(z^{\prime}\right) \varphi\left(z^{\prime}\right) \psi_{\mathbf{k}, s}\left(z^{\prime}\right),
\end{aligned}
$$

где $q=|\mathbf{q}|$.

Используя преобразование Фурье для функции $\varphi(z)$, интегро-дифференциальное уравнение (8) можно преобразовать в интегральное уравнение:

$$
\begin{aligned}
\varphi(z)= & \frac{4 \pi e^{2}}{\kappa S} \int d k_{z} \exp \left(i k_{z} z\right) \sum_{\mathbf{k}, s, s^{\prime}} \frac{g_{s, s^{\prime}}\left(\mathbf{k}, \mathbf{k}+\mathbf{q}, k_{z}\right)}{\left(q^{2}+k_{z}^{2}\right)} \\
& \times \frac{\left(f_{s}(\mathbf{k})-f_{s^{\prime}}(\mathbf{k}+\mathbf{q})\right)}{\varepsilon_{s}(\mathbf{k})+h \omega-\varepsilon_{s^{\prime}}(\mathbf{k}+\mathbf{q})+i h \alpha} \\
& \times \int d z^{\prime} \psi_{\mathbf{k}+\mathbf{q}, s^{\prime}}^{*}\left(z^{\prime}\right) \varphi\left(z^{\prime}\right) \psi_{\mathbf{k}, s}\left(z^{\prime}\right),
\end{aligned}
$$

где $g_{s, s^{\prime}}\left(\mathbf{k}, \mathbf{k}+\mathbf{q}, k_{z}\right)=\int d z \exp \left(-i k_{z} z\right) \psi_{\mathbf{k}, s}^{*}(z) \psi_{\mathbf{k}+\mathbf{q}, s^{\prime}}(z)$ $\times(2 \pi)^{-1 / 2}$. Для нахождения решений (9) сделаем два упрощающих предположения. Первое состоит в том, что мы пренебрежем зависимостью $\int d z^{\prime} \psi_{\mathbf{k}+\mathbf{q}, s^{\prime}}^{*}\left(z^{\prime}\right) \varphi\left(z^{\prime}\right) \psi_{\mathbf{k}, s}\left(z^{\prime}\right)$ от $\mathbf{k}, \mathbf{q}$, а также будем полагать, что этот интеграл отличен от нуля только для $s=s^{\prime}$, причем в каждой подзоне размерного квантования интеграл не зависит от спина электронов. Отметим, что это предположение является точным для случая, когда движения электрона вдоль и поперек квантовой ямы независимы. Численный анализ функций для $\psi_{\mathbf{k}+\mathbf{q}, s^{\prime}}^{*}\left(z^{\prime}\right) \psi_{\mathbf{k}, s}\left(z^{\prime}\right)$ двух рассмотренных далее квантовых ям, в которых $d q \leq 1$ ( $d-$ ширина КЯ), показывает адекватность этих приближений.

Используя это предположение, индексы, соответствующие волновому вектору в интеграле $\int d z^{\prime} \psi_{\mathbf{k}+\mathbf{q}, s^{\prime}}^{*}\left(z^{\prime}\right) \varphi\left(z^{\prime}\right) \psi_{\mathbf{k}, s}\left(z^{\prime}\right)$, можно опустить. 
Второе предположение состоит в том, что спектр вырожден по спину и электронами заполнена только нижняя подзона размерного квантования в зоне проводимости, а дырки имеются только в самой верхней валентной подзоне. Поэтому для зоны проводимости вместо $s$ будем использовать индекс $c$, а для валентной зоны индекс $v$. Умножая (9) сначала на $\psi_{c}^{*}(z) \psi_{c}(z)$ и интегрируя по $z$, а затем выполняя аналогичную операцию с волновыми функциями валентной зоны, получаем систему двух уравнений:

$$
\begin{aligned}
& I_{c}= I_{c} \int d k_{z} \frac{g_{c, c}\left(k_{z}\right) g_{v, v}\left(-k_{z}\right)}{q^{2}+k_{z}^{2}} A_{c}(q, \omega) \\
&+I_{v} \int d k_{z} \frac{g_{v, v}\left(k_{z}\right) g_{c, c}\left(-k_{z}\right)}{q^{2}+k_{z}^{2}} A_{v}(q, \omega) \\
& I_{v}=I_{c} \int d k_{z} \frac{g_{c, c}\left(k_{z}\right) g_{v, v}\left(-k_{z}\right)}{q^{2}+k_{z}^{2}} A_{c}(q, \omega) \\
&+I_{v} \int d k_{z} \frac{g_{v, v}\left(k_{z}\right) g_{v, v}\left(-k_{z}\right)}{q^{2}+k_{z}^{2}} A_{v}(q, \omega),
\end{aligned}
$$

где

$$
\begin{gathered}
I_{j}=\int d z^{\prime} \varphi\left(z^{\prime}\right) \psi_{j}^{*}\left(z^{\prime}\right) \psi_{j}\left(z^{\prime}\right) \\
A_{j}(q, \omega)=\frac{2 e^{2}}{\kappa \pi} \int d^{2} k \frac{\left(f_{j}(\mathbf{k})-f_{j}(\mathbf{k}+\mathbf{q})\right)}{\varepsilon_{j}(\mathbf{k})+h \omega-\varepsilon_{j}(\mathbf{k}+\mathbf{q})+i h \alpha},
\end{gathered}
$$

где $j=c, v$.

Поскольку (10) является системой двух линейных однородных уравнений относительно $I_{c}$ и $I_{v}$, условием существования нетривиального решения является выполнение равенства

$$
\begin{aligned}
& {\left[\int d k_{z} \frac{g_{c, c}\left(k_{z}\right) g_{c, c}\left(-k_{z}\right)}{q^{2}+k_{z}^{2}} A_{c}(q, \omega)-1\right]} \\
& \times\left[\int d k_{z} \frac{g_{v, v}\left(k_{z}\right) g_{v, v}\left(-k_{z}\right)}{q^{2}+k_{z}^{2}} A_{v}(q, \omega)-1\right] \\
& \quad-\int d k_{z} \frac{g_{v, v}\left(k_{z}\right) g_{c, c}\left(-k_{z}\right)}{q^{2}+k_{z}^{2}} A_{v}(q, \omega) \\
& \times \int d k_{z} \frac{g_{c, c}\left(k_{z}\right) g_{v, v}\left(-k_{z}\right)}{q^{2}+k_{z}^{2}} A_{c}(q, \omega)=0,
\end{aligned}
$$

которое представляет собой уравнение для нахождения зависимости частоты плазмона от его волнового вектоpa. В том случае, когда размер локализации волновых функций вдоль оси $z$ много меньше $1 / q$, справедливы соотношения

$$
\begin{gathered}
g_{c}\left(k_{z}\right)=g_{v}\left(k_{z}\right)=(2 \pi)^{-1 / 2}, \\
\int d k_{z} \frac{g_{c}\left(k_{z}\right) g_{c}\left(-k_{z}\right)}{q^{2}+k_{z}^{2}}=\frac{1}{2 q} .
\end{gathered}
$$

В этом случае (12) можно представить в виде

$$
\begin{aligned}
1= & \frac{2 \pi e^{2}}{q \kappa S} \sum_{k}\left[\frac{f_{c}(\mathbf{k})-f_{c}(\mathbf{k}+\mathbf{q})}{\varepsilon_{c}(\mathbf{k})+h \omega-\varepsilon_{c}(\mathbf{k}+\mathbf{q})+i h \alpha}\right. \\
& \left.+\frac{f_{v}(\mathbf{k})-f_{v}(\mathbf{k}+\mathbf{q})}{\varepsilon_{v}(\mathbf{k})+h \omega-\varepsilon_{v}(\mathbf{k}+\mathbf{q})+i h \alpha}\right],
\end{aligned}
$$

что соответствует „обычному“ (т.е. когда ширина квантовой ямы полагается бесконечно малой) дисперсионному уравнению для плазмонов с учетом пространственной дисперсии поляризуемости электронного и дырочного газов [1]. Таким образом, мы показали, что в предельном случае (12) переходит в „обычное“ уравнение для нахождения закона дисперсии двумерного плазмона, в котором учтена пространственная дисперсии поляризуемости электронного газа.

\section{3. Результаты и обсуждение}

Расчет проводился для структур, выращенных на плоскости (013) CdTe, поскольку они наиболее часто используются для наблюдения лазерной генерации [7-9]. Концентрации электронов и дырок полагались одинаковыми, а температура $T=4.2 \mathrm{~K}$. Функции распределения электронов в зонах полагались равными

$$
f_{j}(\mathbf{k})=\left[1+\exp \left(\frac{\varepsilon_{j}(\mathbf{k})-F_{j}}{k_{\mathrm{B}} T}\right)\right]^{-1},
$$

где $k_{\mathrm{B}}$ - постоянная Больцмана. Величины химических потенциалов в зонах находились из условия равенства концентрации электронов и дырок, вычисленных с помощью (15), заданным концентрациям.

Для расчета электронных состояний использовалась модель Кейна с учетом деформационных эффектов. Для простоты пренебрегали понижением симметрии структуры на гетерограницах и отсутствием центра инверсии, что приводило к двукратному вырождению электронного спектра. Детали расчета можно найти в [10]. Рассматривались две квантовые ямы с одинаковой шириной запрещенной зоны (35 мэВ), но с разными ширинами: 5 и 11.75 нм окруженные барьерами $\mathrm{Cd}_{0.7} \mathrm{Hg}_{0.3} \mathrm{Te}$. Материалом для квантовой ямы 5 нм полагался $\mathrm{HgTe}$, а для $11.75 \mathrm{Hм}-\mathrm{Cd}_{0.1} \mathrm{Hg}_{0.9}$ Те. На рис. 1 приведены электронные спектры для этих ям.

На рис. 2 приведены вычисленные зависимости энергии двумерного плазмона от его волнового вектора для квантовой ямы HgTe 5 нм при двух концентрациях неравновесных носителей $n=p=2 \cdot 10^{11}$ и $5 \cdot 10^{11} \mathrm{~cm}^{-2}$. Сплошные кривые были получены с помощью решения уравнения (12), штриховые - с помощью решения уравнения (14). Из рис. 2 видно, что для энергий плазмона $>20$ мэВ зависимость частоты плазмона от волнового вектора близка к линейной $\omega \propto q$. С ростом концентрации носителей растет фазовая скорость плазмона. Учет конечной ширины квантовой ямы приводит к 
уменьшению фазовой скорости плазмона. Для меньших концентраций носителей этот эффект более выражен. Из рис. 2 видно, что учет отличной от нуля ширины квантовой ямы в расчетах аналогичен эффективному уменьшению концентрации носителей в квантовой яме.

Для того чтобы объяснить линейную зависимость энергии плазмона от волнового вектора при больших значениях $q$, заметим, что для больших волновых векторов электрона закон дисперсии электронов в зоне проводимости близок к линейному (см. рис. 1), т.е. при больших волновых векторах зависимость энергии электрона от волнового вектора $\mathrm{k}$ можно представить в виде $\varepsilon_{c}(\mathbf{k}) \approx h V k+\varepsilon_{0}$, где $V$ - максимальная скорость электрона. Кроме того, из рис. 1 видно, что для больших волновых векторов $k$ эффективная масса дырок много больше массы электронов, и поэтому дырочная поляризуемость много меньше электронной. По этой причине
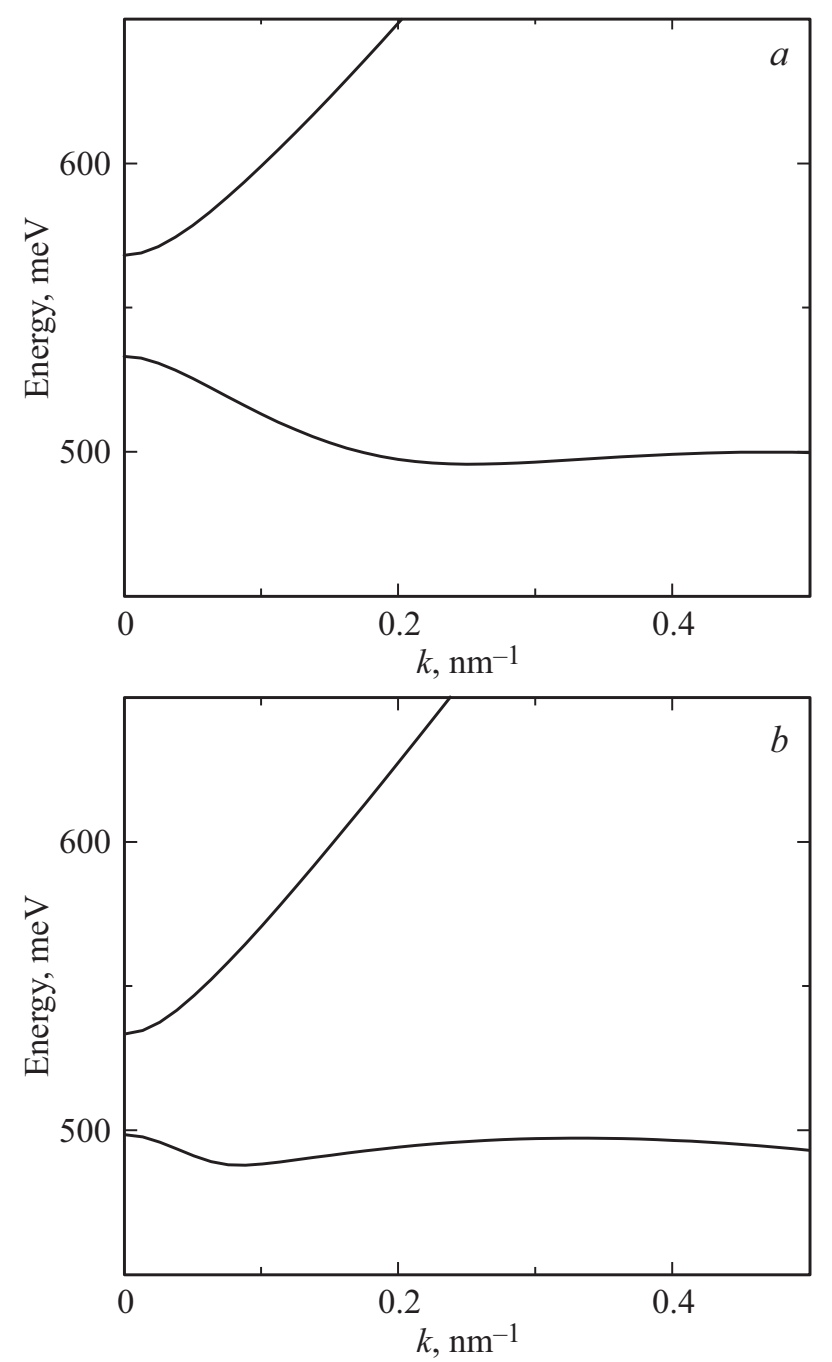

Pис. 1. Спектры электронов в квантовых ямах $\mathrm{HgTe} 5$ нм $(a)$ и $\mathrm{Cd}_{0.1} \mathrm{Hg}_{0.9} \mathrm{Te} 11.75$ нм $(b)$. Волновой вектор направлен вдоль кристаллографического направления [100]. Квантовые ямы выращены на плоскости (013) CdTe и окружены барьерами $\mathrm{Cd}_{0.7} \mathrm{Hg}_{0.3} \mathrm{Te}$. Температура $4.2 \mathrm{~K}$.

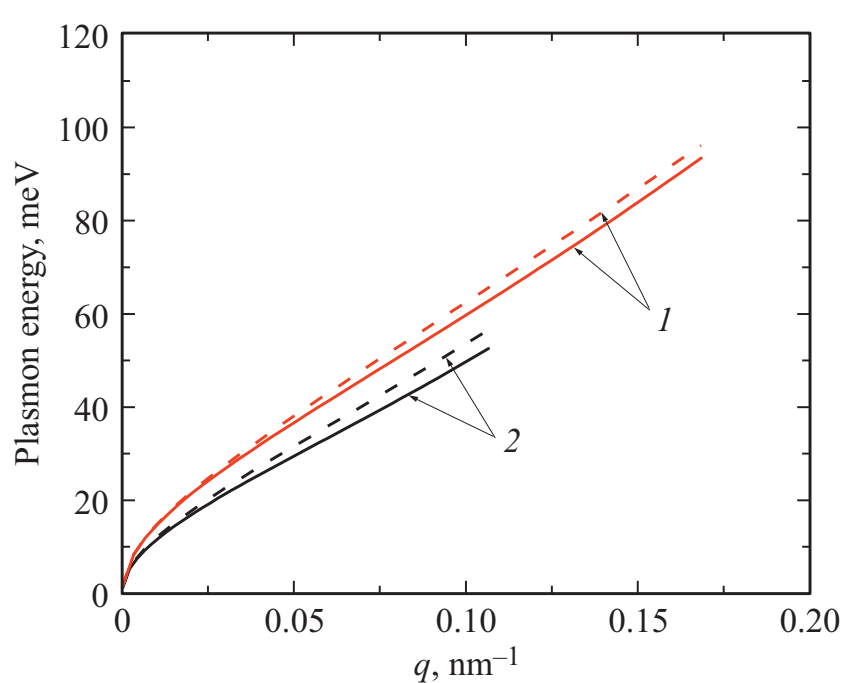

Рис. 2. Зависимости энергии плазмона от волнового вектора в квантовой яме $\mathrm{HgTe} 5$ нм. Концентрации электронов и дырок, $\mathrm{cm}^{-2}: 1-5 \cdot 10^{11}, 2-2 \cdot 10^{11} \mathrm{~cm}^{-2}$. Сплошные линии результат решения уравнения (12), штриховые - (14).

закон дисперсии плазмонов в основном определяется электронами и вторым слагаемым в правой части (14) можно пренебречь. Если $q \gg k_{\mathrm{F}}$, где $k_{\mathrm{F}}-$ волновой вектор на уровне Ферми, то правую часть (14) можно представить в виде

$$
\begin{aligned}
\frac{2 \pi e^{2}}{q \kappa S} \sum_{k} & {\left[\frac{f_{c}(\mathbf{k})-f_{c}(\mathbf{k}+\mathbf{q})}{\varepsilon_{c}(\mathbf{k})+h \omega-\varepsilon_{c}(\mathbf{k}+\mathbf{q})+i h \alpha}\right] } \\
& \approx \frac{2 \pi e^{2} n}{\kappa} \frac{h V}{(h \omega)^{2}-(h V q)^{2}}
\end{aligned}
$$

где $n$ - концентрация электронов в квантовой яме. Подставляя (16) в (14), находим

$$
h \omega \approx \sqrt{(h V q)^{2}+\frac{2 \pi e^{2} n h V}{\kappa}} .
$$

Из (17) видно, что при больших $q$ частота плазмона линейно зависит от волнового вектора $\omega \sim q$, а групповая скорость плазмона стремится к максимальной скорости электрона. Отметим, что расчеты спектра в графене дают аналогичную картину [4].

На рис. 3 приведены вычисленные зависимости энергии двумерного плазмона от его волнового вектора для квантовой ямы $\mathrm{Cd}_{0.1} \mathrm{Hg}_{0.9} \mathrm{Te} 11.75 \mathrm{Hм}$, окруженной барьерами $\mathrm{Cd}_{0.7} \mathrm{Hg}_{0.3} \mathrm{Te}$. Эта яма имеет такую же ширину запрещенной зоны, что и квантовая яма $\mathrm{HgTe},-5$ нм. Добавление кадмия в квантовую яму, при сохранении величины запрещенной зоны, увеличивает ее толщину. Поэтому учет ширины квантовой ямы при вычислении закона дисперсии в этом случае сильнее влияет за зависимость энергии плазмона от волнового вектора. Из сравнения рис. 2 и 3 видно, что добавление кадмия и 


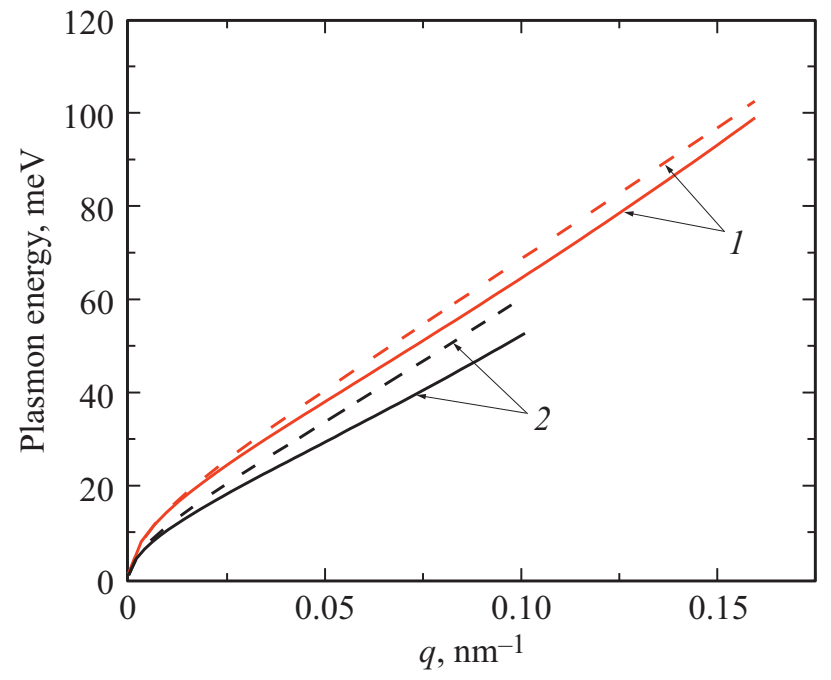

Рис. 3. Зависимость энергии плазмона от волнового вектора в квантовой яме $\mathrm{Cd}_{0.1} \mathrm{Hg}_{0.9} \mathrm{Te} 11.75$ нм. Концентрации электронов и дырок, $\mathrm{cm}^{-2}: 1-5 \cdot 10^{11}, 2-2 \cdot 10^{11} \mathrm{~cm}^{-2}$. Сплошные линии - результат решения уравнения (12), штриховые - (14).

увеличение толщины квантовой ямы при сохранении ширины запрещенной зоны немного увеличивает скорость плазмона. Из рис. 3 также видно, что для концентрации электронов и дырок $2 \cdot 10^{11} \mathrm{~cm}^{-2}$ уменьшение энергии плазмона, вследствие учета конечной ширины КЯ, при определенном волновом векторе на линейном участке дисперсионной кривой примерно вдвое больше, чем для концентрации $5 \cdot 10^{11} \mathrm{~cm}^{-2}$.

\section{4. Заключение}

В заключение приведем основные результаты работы. Показано, что частотная дисперсия поляризуемости носителей тока в квантовой яме приводит при больших векторах двумерного плазмона к зависимости $\omega \propto q$. Групповая скорость плазмона при больших $q$ стремится к максимальной скорости электрона в нижней подзоне размерного квантования. При заданном волновом векторе учет ширины квантовой ямы при вычислении закона дисперсии приводит к уменьшению энергии плазмона, аналогичному уменьшению энергии плазмона, происходящему при уменьшении концентрации носителей заряда в квантовой яме.

\section{Финансирование работы}

Работа выполнена при финансовой поддержке Российского научного фонда (грант № 20-42-09039).

\section{Конфликт интересов}

Авторы заявляют, что у них нет конфликта интересов.

\section{Список литературы}

[1] F. Stern. Phys. Rev. Lett., 18, 546 (1967).

[2] B. Jusserand, D. Richards, B. Etienne, H. Peric, G. Fasol. Surf. Sci., 263, 527 (1992).

[3] K. Kapralov, G. Alymov, D. Svintsov, A.A. Dubinov. J. Phys. Condens. Matter, 32, 065301 (2020).

[4] F. Rana, J.H. Strait, H. Wang, C. Manolatou. Phys. Rev. B, 84, 045437 (2011).

[5] V.A. Volkov, S.A. Mikhailov. Modern Problems in Condensed Matter Sci., 27, 855 (1991).

[6] Дж. Займан. Принципы теории твердого тела (М., Мир, 1966) гл. 5, с. 170.

[7] S.V. Morozov, V.V. Rumyantsev, M.A. Fadeev, M.S.Zholudev, K.E. Kudryavtsev, A.V. Antonov, A.M. Kadykov, A.A. Dubinov, N.N. Mikhailov, S.A. Dvoretsky, V.I. Gavrilenko. Appl. Phys. Lett., 111, 192101 (2017).

[8] K.E. Kudryavtsev, V.V. Rumyantsev, V.Ya. Aleshkin, A.A. Dubinov, V.V. Utochkin, M.A. Fadeev, N.N. Mikhailov, G. Alymov, D. Svintsov, V.I. Gavrilenko, S.V. Morozov. Appl. Phys. Lett., 117, 083103 (2020).

[9] S.V. Morozov, V.V. Rumyantsev, A.M. Kadykov, A.A. Dubinov, K.E. Kudryavtsev, A.V. Antonov, N.N. Mikhailov, S.A. Dvoretskii, V.I. Gavrilenko. Appl. Phys. Lett., 108, 092104 (2016).

[10] G.M. Minkov, V.Ya. Aleshkin, O.E. Rut, A.A. Sherstobitov, A.V. Germanenko, S.A. Dvoretski, N.N. Mikhailov. Phys. Rev. B, 96, 035320 (2017).

Редактор Л.В. Шаронова

\section{Influence of quantum well parameters on the spectrum of two-dimensional plasmons in $\mathrm{HgTe} / \mathrm{CdHgTe}$ heterostructures}

\section{V.Ya. Aleshkin 1,2, A.A. Dubinov ${ }^{1,2}$}

${ }^{1}$ Institute for Physics of Microstructures, Russian Academy of Sciences, 603950 Nizhny Novgorod, Russia 2 Lobachevsky State University of Nizhny Novgorod, 603950 Nizhny Novgorod, Russia

Abstract Influence of parameters of a quantum well and electronic polarizability spatial dispersion on the dependence of two-dimensional plasmon energy on wave vector in narrow-gap $\mathrm{CdHgTe}$ quantum wells (band gap $35 \mathrm{meV}$ ) is studied theoretically. It is shown that at energies above $20 \mathrm{meV}$, the dispersion law of two-dimensional plasmons is close to linear. Taking into account the finite width of the quantum well decreases the plasmon phase velocity. This effect increases with an increase in the fraction of cadmium in the QW while maintaining the band gap and with a decrease in the concentration of charge carriers in it. 